\title{
Simulation of binary mixtures condensation using higher order methods
}

\author{
H. Deng, M. Fernandino \& C. A. Dorao \\ Norwegian University of Science and Technology, Norway
}

\begin{abstract}
The use of refrigerant mixtures as good substitutes to the phased-out pure refrigerants has motivated the study of condensation heat and mass transfer of mixtures in the recent years. This work presents a one-dimensional steadystate non-equilibrium model to study the condensation heat and mass transfer characteristics of binary mixtures inside horizontal smooth tubes. The leastsquares finite element method (LSFEM) with higher order approximations shows larger convergence rate when solving the system of equations. The results are compared with experimental data and show a reasonably good prediction of the heat transfer coefficients and fluid temperatures. It is also found that the mass transfer resistance in the vapor phase is significant to the mixture condensation process, which may not be neglected in both experimental and analytical studies. The sensible heat transfer resistance in vapor is not very important and can be neglected.

Keywords: in-tube condensation, non-azeotropic refrigerant mixtures, heat and mass transfer resistances, heat transfer coefficient, higher order methods.
\end{abstract}

\section{Introduction}

The non-azeotropic refrigerant mixtures are good substitutes of chlorofluorocarbons (CFCs) and hydrochlorofluorocarbons (HCFCs) as they have zero ozone depleting potential (ODP) and low global warming potential (GWP). The study on condensation of non-azeotropic refrigerant mixtures in horizontal tubes becomes more attractive in order to develop the suitable working fluids in heat exchangers.

During the in-tube condensation of non-azeotropic refrigerant mixtures the less volatile component condenses preferentially which causes the lower local saturation temperature, as well as a composition shift in both liquid and vapor 
phases. The temperature and composition differences between the vapor and vapor-side interface, the liquid and liquid-side interface result in heat and mass transfer resistances. For these phenomena that differ from that of pure refrigerant condensation, the heat and mass transfer characteristics of mixture condensation processes should be studied specifically. Meanwhile, effective and efficient computational methods are important for the simulation of the problem.

Both experimental and theoretical work have been done for in-tube condensation of mixtures, and a review on non-azeotropic fluid mixtures has been presented by Fronk and Garimella [1]. Among the theoretical studies, Koyama et al. [2] proposed a prediction model considering the mass transfer resistance in vapor phase and assuming the vapor saturated. Jin et al. [3] developed the model by considering the mass transfer resistances in both phases. These two models are widely used in experimental works for comparison.

The present work aims at studying the heat and mass transfer characteristics during mixture condensation process with emphases on the resistances in the vapor phase, by removing the assumption of saturated vapor. A higher order method is employed to solve the system of equations. The physical and mathematical models are described in Section 2 and the LSFEM is presented in Section 3. The convergence analysis regarding the higher order approximations is made in Section 4. Section 5 presents the calculated results and discussions. Finally, the main conclusions are summarised in Section 6.

\section{Model description}

The physical model is shown in fig. 1. The less volatile component condenses more readily, causing a lower concentration of the more volatile component in the bulk vapor than vapor-side interface. The composition difference results in the mass transfer diffusion. The temperature difference between the vapor and interface causes the sensible heat transfer resistance.

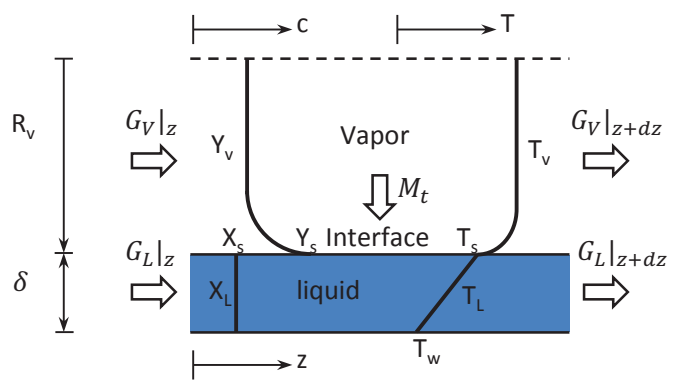

Figure 1: Physical model.

The assumptions for calculation of the mass and heat transfer of mixture condensation are: 
1. The liquid and vapor are locally at the same pressure and the interface is in thermodynamic equilibrium.

2. The liquid phase is well mixed and the mass transfer resistance in liquid phase is neglected.

3. The vapor phase can be either saturated or superheated.

4. The flow is annular and the film thickness is circumferentially uniform.

\subsection{Conservation equations}

\subsubsection{Two-phase flow}

Under these assumptions, a one-dimension steady-state non-equilibrium model is expressed as follows. The mass, species (for the more volatile component) and energy conservation equations for the vapor phase can be written as

$$
\begin{gathered}
\frac{\mathrm{d}}{\mathrm{d} z}\left(\alpha \rho_{\mathrm{v}} V_{\mathrm{v}}\right)=-M_{\mathrm{t}} \\
\frac{\mathrm{d}}{\mathrm{d} z}\left(\alpha \rho_{\mathrm{v}} V_{\mathrm{v}} Y\right)=-M_{1} \\
\frac{\mathrm{d}}{\mathrm{d} z}\left(\alpha \rho_{\mathrm{v}} V_{\mathrm{v}} i_{\mathrm{v}}\right)-i_{\mathrm{vs}} \frac{\mathrm{d}}{\mathrm{d} z}\left(\alpha \rho_{\mathrm{v}} V_{\mathrm{v}}\right)=-\frac{4 D_{\mathrm{v}}}{D_{\mathrm{w}}^{2}} Q_{\mathrm{sv}}
\end{gathered}
$$

where the subscript "v" stands for vapor phase, "vs" for vapor-side interface, "w" for wall and " 1 " for the more volatile component, $\alpha$ is the vapor fraction, $z[\mathrm{~m}]$ is the axial space coordinate, $\rho\left[\mathrm{kg} / \mathrm{m}^{3}\right]$ is density, $V[\mathrm{~m} / \mathrm{s}]$ is velocity, $Y$ is the composition of component 1 in vapor phase, $M_{\mathrm{t}}\left[\mathrm{kg} / \mathrm{m}^{3} \mathrm{~s}\right]$ is the total condensation rate, $M_{1}\left[\mathrm{~kg} / \mathrm{m}^{3} \mathrm{~s}\right]$ is the condensation rate of component $1, i[\mathrm{~J} / \mathrm{kg}]$ is the specific enthalpy, $D[\mathrm{~m}]$ is diameter and $Q\left[\mathrm{~W} / \mathrm{m}^{2}\right]$ is the heat flux.

The corresponding equations for the liquid phase are

$$
\begin{gathered}
\frac{\mathrm{d}}{\mathrm{d} z}\left((1-\alpha) \rho_{\mathrm{l}} V_{\mathrm{l}}\right)=M_{\mathrm{t}} \\
\frac{\mathrm{d}}{\mathrm{d} z}\left((1-\alpha) \rho_{\mathrm{l}} V_{\mathrm{l}} X\right)=M_{1} \\
\frac{\mathrm{d}}{\mathrm{d} z}\left((1-\alpha) \rho_{\mathrm{l}} V_{\mathrm{l}} i_{\mathrm{l}}\right)-i_{\mathrm{ls}} \frac{\mathrm{d}}{\mathrm{d} z}\left((1-\alpha) \rho_{\mathrm{l}} V_{\mathrm{l}}\right)=\frac{4 D_{\mathrm{v}}}{D_{\mathrm{w}}^{2}}\left(Q_{\mathrm{lv}}+Q_{\mathrm{sv}}\right)-\frac{4}{D_{\mathrm{w}}} Q_{\mathrm{w}} .
\end{gathered}
$$

where $X$ is the composition of component 1 in liquid phase, and the subscript "l" stands for liquid phase.

The combined momentum equation for the vapor and liquid phase is given by

$$
\frac{\mathrm{d}}{\mathrm{d} z}\left(\alpha \rho_{\mathrm{v}} V_{\mathrm{v}}^{2}\right)+\frac{\mathrm{d}}{\mathrm{d} z}\left((1-\alpha) \rho_{\mathrm{l}} V_{\mathrm{l}}^{2}\right)=-\frac{\mathrm{d} P}{\mathrm{~d} z}-\frac{4}{D_{\mathrm{w}}} \tau_{\mathrm{w}}
$$

where $P[\mathrm{~Pa}]$ is the pressure and $\tau_{\mathrm{w}}\left[\mathrm{N} / \mathrm{m}^{2}\right]$ is the wall shear stress. 


\subsection{Constitutive equations}

\subsubsection{Heat flux balance}

From the heat balance of refrigerant, the wall heat flux is equal to the sum of latent heat $Q_{\mathrm{lv}}$ and the vapor sensible heat $Q_{\mathrm{sv}}$,

$$
\begin{gathered}
Q_{\mathrm{w}}=h_{\mathrm{l}}\left(T_{\mathrm{s}}-T_{\mathrm{w}}\right)=Q_{\mathrm{lv}}+Q_{\mathrm{sv}} \\
Q_{\mathrm{lv}}=\frac{D_{\mathrm{w}}^{2}}{4 D_{\mathrm{v}}} M_{\mathrm{t}} i_{\mathrm{lv}} \\
Q_{\mathrm{sv}}=h_{\mathrm{v}}\left(T_{\mathrm{v}}-T_{\mathrm{s}}\right) .
\end{gathered}
$$

where $i_{\mathrm{lv}}[\mathrm{J} / \mathrm{kg}]$ is the enthalpy of vaporization calculated at the interface temperature $T_{\mathrm{s}}[\mathrm{K}]$, and $T_{\mathrm{v}}[\mathrm{K}]$ is the vapor bulk temperature.

\subsubsection{Condensation rates}

The condensation rate of the more volatile component is made up of convective and diffusion contributions:

$$
M_{1}=M_{\mathrm{t}} Y_{\mathrm{s}}-\frac{4 D_{\mathrm{v}}}{D_{\mathrm{w}}^{2}} k_{\mathrm{v}}\left(Y_{\mathrm{s}}-Y\right)
$$

where $Y_{\mathrm{s}}$ is the composition of component 1 at vapor-side interface, $k_{\mathrm{v}}$ is the mass transfer coefficient calculating by the correlation of Linton and Sherwood [4].

\subsubsection{Liquid, vapor and interface compositions, and mixture properties}

The vapor quality $x$ and compositions in the liquid and vapor phase satisfies the species conservation

$$
x=\frac{C_{0}-X}{Y-X}
$$

where $C_{0}$ denotes the initial composition. As thermodynamic equilibrium at the interface is assumed, the interface temperature and the composition interface can be computed by the equation of state. The thermodynamic and transport properties of the refrigerant mixtures, which are pressure, temperature and composition dependent, are calculated using the REFPROP database [5].

\subsubsection{Friction factor}

In this work, Friedel's [6] correlation is used. The two-phase multiplier $\Phi_{\mathrm{lo}}^{2}$ is obtained from

$$
\Phi_{\mathrm{lo}}^{2}=(1-x)^{2}+x^{2} \frac{\rho_{1} f_{\mathrm{vo}}}{\rho_{\mathrm{v}} f_{\mathrm{lo}}}+3.24 A_{2} A_{3} F r^{-0.045} W e^{-0.035}
$$

with

$$
A_{2}=x^{0.78}(1-x)^{0.224}, \quad A_{3}=\left(\frac{\rho_{1}}{\rho_{\mathrm{v}}}\right)^{0.91}\left(\frac{\mu_{\mathrm{v}}}{\mu_{\mathrm{l}}}\right)^{0.19}\left(1-\frac{\mu_{\mathrm{v}}}{\mu_{\mathrm{l}}}\right)^{0.7}
$$

where $\mathrm{Fr}$ is Froude number, $W e$ is Weber number and $\mu[\mathrm{Pa} \cdot \mathrm{s}]$ is the dynamic viscosity. The friction factor $f_{\mathrm{lo}}, f_{\mathrm{vo}}$ is computed from Haaland's [7] equation. 
Then, the wall shear stress can be given as,

$$
\tau_{\mathrm{w}}=f_{\mathrm{lo}} \frac{G^{2}}{2 \rho_{\mathrm{l}}} \Phi_{\mathrm{lo}}^{2} .
$$

where $G\left[\mathrm{~kg} / \mathrm{m}^{2} \mathrm{~s}\right]$ is the mass flux.

\subsubsection{Void fraction and diameter of vapor core}

The void fraction $\alpha$ can be simply calculated from Chisholm's [8] correlation:

$$
\alpha=\left[1-x\left(1-\frac{\rho_{\mathrm{l}}}{\rho_{\mathrm{v}}}\right)\right]^{1 / 2} .
$$

The vapor core diameter $D_{\mathrm{v}}$ is determined from

$$
D_{\mathrm{v}}=D_{\mathrm{w}}-2 \delta=D_{\mathrm{w}} \alpha^{0.5}
$$

where $\delta[\mathrm{m}]$ is the thickness of liquid film.

\subsubsection{Heat transfer coefficients}

The heat transfer coefficient of the vapor phase is given by the Dittus-Boelter correlation, and the heat transfer coefficient of the liquid film is computed from Dobson and Chato's [9] correlation

$$
\begin{gathered}
h_{\mathrm{v}}=0.023 \operatorname{Re}_{\mathrm{v}}{ }^{0.8} \operatorname{Pr}_{\mathrm{v}}{ }^{0.4} \lambda_{\mathrm{v}} / \mathrm{D}_{\mathrm{v}} \\
h_{\mathrm{l}}=0.023 \operatorname{Re}_{\mathrm{l}}{ }^{0.8} \operatorname{Pr}_{\mathrm{l}}{ }^{0.4}\left(1+\frac{2.22}{X_{\mathrm{tt}}^{0.89}}\right) \frac{\lambda_{\mathrm{l}}}{\mathrm{D}_{\mathrm{w}}}
\end{gathered}
$$

where $\lambda[\mathrm{W} / \mathrm{mK}]$ is the thermal conductivity, and $X_{\mathrm{tt}}$ is the Martinelli parameter.

The total heat transfer coefficient is defined as

$$
h_{\mathrm{t}}=\frac{Q_{\mathrm{w}}}{T_{\mathrm{v}}-T_{\mathrm{w}}}
$$

\section{The least-squares finite element method}

In the general case the least-squares formulation [10] is based on the minimization of a norm-equivalent functional. For simplicity, the system of equations can be represented as

$$
\begin{array}{ll}
\mathcal{L} \mathbf{u}=\mathbf{g} & \text { in } \Omega \\
\mathcal{B} \mathbf{u}=\mathbf{u}_{\mathrm{d} \Omega} & \text { on } \mathrm{d} \Omega
\end{array}
$$

with $\mathcal{L}$ a linear partial differential operator and $\mathrm{B}$ the trace operator. Here, it is assumed that the system is well-posed and the operator $(\mathcal{L}, \mathcal{B})$ is a continuous mapping between the function space $X(\Omega)$ onto the space $Y(\Omega) \times Y(\mathrm{~d} \Omega)$. 
The norm equivalent functional becomes

$$
\mathcal{J}(\mathbf{u}) \equiv \frac{1}{2}\|\mathcal{L} \mathbf{u}-\mathbf{g}\|_{Y(\Omega)}^{2}+\frac{1}{2}\left\|\mathcal{B} \mathbf{u}-\mathbf{u}_{\mathrm{d} \Omega}\right\|_{Y(\mathrm{~d} \Omega)}^{2} .
$$

Based on variational analysis, the minimization statement is equivalent to:

$$
\lim _{\epsilon \rightarrow 0} \frac{d}{d \epsilon} \mathcal{J}(\mathbf{u}+\epsilon \mathbf{v})=0 \quad \forall \mathbf{u} \in X(\Omega) .
$$

Hence, the necessary condition for the minimization of $\mathcal{J}$ is equivalent to find $\mathbf{u} \in X(\Omega)$ such that

$$
\mathcal{A}(\mathbf{u}, \mathbf{v})=\mathcal{F}(\mathbf{v}) \quad \forall \mathbf{v} \in X(\Omega)
$$

with

$$
\begin{gathered}
\mathcal{A}(\mathbf{u}, \mathbf{v})=\langle\mathcal{L} \mathbf{u}, \mathcal{L} \mathbf{v}\rangle_{Y(\Omega)}+\langle\mathcal{B} \mathbf{u}, \mathcal{B} \mathbf{v}\rangle_{Y(\mathrm{~d} \Omega)} \\
\mathcal{F}(\mathbf{v})=\langle\mathbf{v}, \mathcal{L} \mathbf{v}\rangle_{Y(\Omega)}+\left\langle\mathbf{u}_{\mathrm{d} \Omega}, \mathcal{B} \mathbf{v}\right\rangle_{Y(\mathrm{~d} \Omega)}
\end{gathered}
$$

where $\mathcal{A}: X \times X \rightarrow \mathbb{R}$ is a symmetric, continuous bilinear form, and $\mathcal{F}: X \rightarrow \mathbb{R}$ is a continuous linear form.

The least-squares residual is denoted as $\mathcal{R}_{L S}$, given by

$$
\mathcal{R}_{L S}(\mathbf{u})=\sqrt{\langle\mathcal{L} \mathbf{u}-\mathbf{g}, \mathcal{L} \mathbf{u}-\mathbf{g}\rangle} .
$$

The introduction of the boundary residual allows the use of spaces $X(\Omega)$ that are not constrained to satisfy the boundary conditions. If the boundary terms are omitted, the boundary conditions must be enforced strongly in the definition of the space $X(\Omega)$.

Finally, the searching space is restricted to a finite dimensional space and the problem becomes: find $\mathbf{u}_{h} \in X_{h}(\Omega) \subset X(\Omega)$ such that

$$
\mathcal{A}\left(\mathbf{u}_{h}, \mathbf{v}_{h}\right)=\mathcal{F}\left(\mathbf{v}_{h}\right) \quad \forall \mathbf{v}_{h} \in X_{h}(\Omega) .
$$

Then, the residual can be computed as

$$
\mathcal{R}_{L S}\left(\mathbf{u}_{h}\right)=\sqrt{\left\langle\mathcal{L} \mathbf{u}_{h}-\mathbf{g}, \mathcal{L} \mathbf{u}_{h}-\mathbf{g}\right\rangle}=\left(\int_{\Omega}\left(\mathcal{L} \mathbf{u}_{h}-\mathbf{g}\right)^{2} \mathrm{~d} \Omega\right)^{1 / 2}
$$

\subsection{Spectral element approximation}

The computational domain $\Omega$ is divided into $N_{e}$ non-overlapping sub-domains $\Omega_{e}$ of diameter $l_{e}$, called spectral elements, such that

$$
\Omega=\bigcup_{e=1}^{N_{e}} \Omega_{e}, \quad \Omega_{e} \cap \Omega_{l}=\varnothing \quad \text { if } e, l \in N_{e}, e \neq l .
$$

Each general element is transformed to a reference element $[-1,1]$ by a continuous, differentiable, one-to-one mapping. Within each element, the local 
solution $\mathbf{u}_{h}^{e}$ can be approximated as

$$
\mathbf{u}_{h}^{e}(z)=\sum_{i=0}^{E} u_{i}^{e} \phi_{i}(\xi), \quad \text { with } u_{i}^{e}=u\left(\xi_{i}\right), \xi_{i} \in[-1,1]
$$

where $E$ is number of degrees of freedom of element $e$, and $\phi_{i}(\xi)$ is the one dimensional basis function. In this work, the basis functions used are Lagrangian polynomials through Gauss-Lobatto-Legendre (GLL) points.

The global approximation in $\Omega, \mathbf{u}_{h}$, is constructed by gluing the local approximations $\mathbf{u}_{h}^{e}$, i.e.

$$
\mathbf{u}_{h}=\bigcup_{e=1}^{N_{e}} \mathbf{u}_{h}^{e}
$$

\section{Convergence analysis}

\subsection{Linearized system}

In order to test the LSFEM, the governing equations eqns. (1)-(3) are expressed in the system of the form eqn. (20) with the boundary conditions of the form eqn. (21). By implementing the Picard iteration, the nonlinear system of equations can be linearized to the system with the components:

$$
\begin{gathered}
\mathcal{L}_{\mathrm{tp}}=\left(\begin{array}{ccc}
G \frac{\mathrm{d}}{\mathrm{d} z} & \mathbf{0} & \mathbf{0} \\
\mathbf{0} & (x G)^{*} \frac{\mathrm{d}}{\mathrm{d} z}+4 D_{\mathrm{v}} k_{\mathrm{v}} / D_{\mathrm{w}}^{2} & \mathbf{0} \\
\mathbf{0} & \mathbf{0} & \left(x c_{p} G\right)^{*} \frac{\mathrm{d}}{\mathrm{d} z}+4 D_{\mathrm{v}} h_{\mathrm{v}} / D_{\mathrm{w}}^{2}
\end{array}\right) \\
\mathbf{u}_{\mathrm{tp}}=\left(\begin{array}{c}
x \\
Y \\
T_{\mathrm{v}}
\end{array}\right) \\
\mathbf{g}_{\mathrm{tp}}=\left(\begin{array}{c}
-M_{\mathrm{t}} \\
-\left(M_{\mathrm{t}}-4 D_{\mathrm{v}} k_{\mathrm{v}} / D_{\mathrm{w}}^{2}\right) Y_{\mathrm{s}} \\
4 D_{\mathrm{v}} h_{\mathrm{v}} T_{\mathrm{s}} / D_{\mathrm{w}}^{2}-i_{\mathrm{vs}} M_{\mathrm{t}}
\end{array}\right)
\end{gathered}
$$

where superscript $*$ means that the variable is known from last iteration.

\subsection{Convergence rate}

The coefficients in the linearized system eqns. (33)-(35) are set as constant to easily test the LSFEM accuracy with different orders of polynomials. The residuals are plotted against the numbers of degrees of freedom, as shown in fig. 2. It can be seen that when using higher order, the smaller residual of the system can be 
achieved at the lower numbers of degrees of freedom. This residual indicates how big the error of the numerical solution is. Additionally, it can be observed that the solution converges faster with the increase of the order $p$. In this specific case, the convergence rates are proportional to the order $p$.

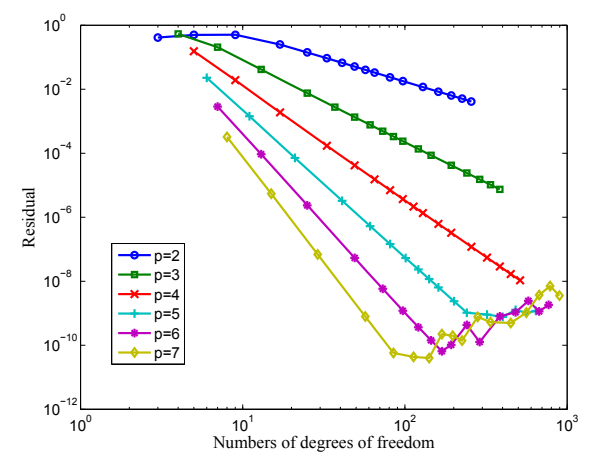

Figure 2: LSFEM convergence analysis.

\section{Results and discussions}

The LSFEM with higher order polynomials is then applied to the present model with real physical properties and working conditions. The results are compared with Kogawa's [11] experimental data. The experiment is for the mixture condensation in a tube of diameter $8.4 \mathrm{~mm}$. The data used here is corresponding to the case with inlet pressure $495 \mathrm{kPa}$, initial composition $0.349 / 0.651 \mathrm{R} 134 \mathrm{a} / \mathrm{R} 123$ by mass fraction, mass flux $300.5 \mathrm{~kg} / \mathrm{m}^{2} \mathrm{~s}$. The wall heat flux applied is interpolated from the experimental data with quadratic polynomials. The inlet temperature is set as superheated as the experimental condition, while Jin et al.'s model [3] mentioned before can be only applied to the saturated vapor.

Figure 3 shows the variations of temperatures, compositions and the ratio of the condensation rate of the more volatile component (R134a in this case) to the total condensation rate $M_{1} / M_{\mathrm{t}}$ along the tube axial position. The predicted and experimental values of wall temperature are compared in fig. 3(a), and they show good agreement generally, except for slight overestimation at the regions near the inlet and outlet of the condenser. Moreover, it can be seen that the predicted temperature of bulk vapor is always higher than that of the interface, and both decrease gradually with the condensation process. The saturated vapor temperature obtained from the equation of state is slightly higher than the interface temperature and they reach almost the same value at the outlet. Under this circumstance the sensible heat transfer resistance can be neglected, as assumed in most of previous studies. However, it turns out in the present study that the bulk vapor is superheated by around $3 \mathrm{~K}$ during the whole process, and the effect of sensible heat transfer 
resistance should be examined. Based on the local quality and phase equilibrium diagram, the calculated thermodynamic equilibrium temperature lies between the interface and saturated vapor temperatures, which is close to saturated vapor temperature in the upstream region while closer to the interface temperature in the downstream region.

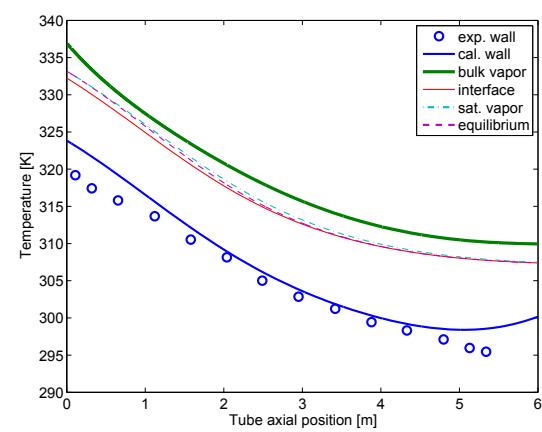

(a)

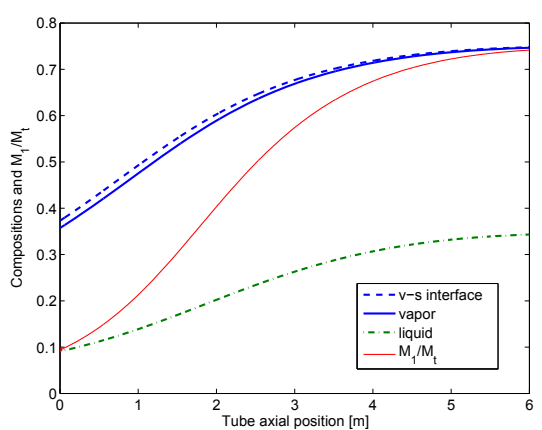

(b)

Figure 3: Variations along the tube axial coordinates: (a) temperatures; (b) compositions and ratio $M_{1} / M_{\mathrm{t}}$.

Following the way of presentation used in Jin et al. [3], the variations of composition of vapor, vapor-side interface and liquid for R134a are presented in fig. 3(b), and all of them increase along the tube axial position. As expected the composition of the more volatile component R134a at vapor-side interface is higher than that of the bulk vapor and the difference vanishes at the outlet. Consequently it results in the higher saturated temperature of vapor than the temperature of the interface, as shown in fig. 3(a). The ratio of the condensation rate of $\mathrm{R} 134 \mathrm{a}$ to the total condensation rate $M_{1} / M_{\mathrm{t}}$ increases with the condensation process. When condensation starts, the less volatile component R123 condenses more readily. It results in large difference in composition of R134a between the vapor-interface and vapor, so that mass diffusion occurs. With the reduction of composition difference, the mass diffusion in the vapor phase decreases. Therefore, the ratio of condensation rate increases and finally reaches $Y_{\mathrm{S}}$ at the outlet. Jin et al.'s model [3] considering mass transfer resistances in both vapor and liquid also gave similar results qualitatively.

Figure 4 presents the variation of the heat transfer coefficient against the quality, and summarises the effects of heat and mass transfer resistance in the vapor phase. The calculated heat transfer coefficient is compared with the experimental data and shows reasonably good prediction, as shown in fig. 4(a). The heat transfer coefficient decreases gradually with the reduction of vapor quality. Also, the heat transfer coefficients calculated based on the thermodynamic equilibrium temperature and saturated vapor temperature are plotted. It clearly shows that in 
the present work using the real bulk vapor temperature gives better estimation of the total heat transfer coefficient than using the other two temperatures, especially at higher quality. Therefore, the calculation for the real temperature of bulk vapor is recommended for better prediction of the heat transfer coefficient.

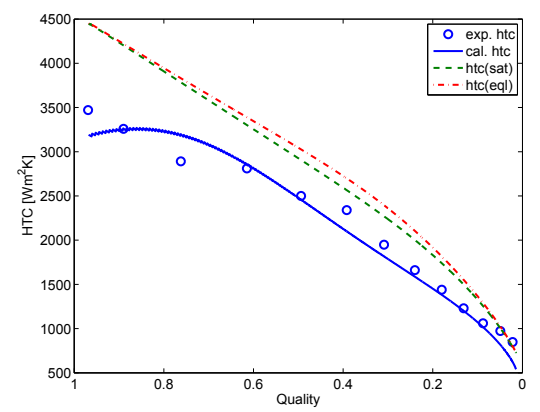

(a)

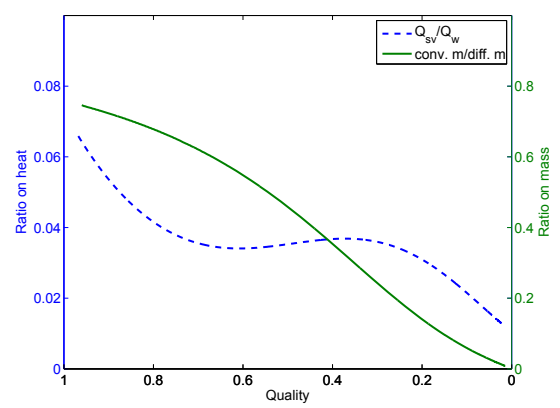

(b)

Figure 4: Variations against quality: (a) heat transfer coefficient; (b) effects of heat and mass transfer resistance.

In order to show the effects of heat and mass transfer resistance in the bulk vapor, the variations of two parameters are illustrated in fig. 4(b). One parameter is the ratio of sensible vapor heat flux to the wall heat flux, $Q_{\mathrm{sv}} / Q_{\mathrm{w}}$, and the other one is the ratio of mass diffusion rate to convection rate for the more volatile component, $\frac{4 D_{\mathrm{v}}}{D_{\mathrm{v}}^{2}} k_{\mathrm{v}}\left(Y_{\mathrm{s}}-Y\right) / M_{\mathrm{t}} Y_{\mathrm{s}}$. The ratio of sensible heat flux to wall heat flux decreases with the condensation process due to the reduction of the vapor heat transfer coefficient. The heat and mass transfer are strongly coupled during the mixtures condensation process. For a given wall heat flux, if the sensible heat flux increases, the latent heat flux becomes smaller so that the mass transfer rate decreases, which in turn affects the heat transfer flux again. In this study, the ratio of sensible heat flux to wall heat flux is smaller than $7 \%$ which means that the sensible heat transfer resistance in vapor can be neglected.

The ratio of mass diffusion to convection also decreases with the increase of quality. It can be seen that the ratio keeps at high level even when the composition difference between vapor-side interface and bulk vapor is small (fig. 3(b)). The mass diffusion term retards significantly the mass transfer rate of the more volatile component, and it also influences the heat transfer rate simultaneously, especially when the difference of specific latent heat between the two components is large.

\section{Conclusions}

The in-tube condensation for a non-azeotropic binary mixture has been studied numerically. The model considering heat and mass transfer resistance in the vapor phase was presented. The system of equations is solved with the least-squares finite 
element method. The method with higher order approximations showed larger convergence rate when solving the simplified system of equations.

The calculated results were compared with Kogawa's [11] experimental data and showed reasonably good agreement. The results also showed that the bulk vapor can be in superheated state during the condensation process, and in the present work using the real bulk vapor temperature gave a better estimation of the heat transfer coefficient than using saturated vapor temperature or system thermodynamic equilibrium temperature. Even though the composition difference of the more volatile component between the vapor-side interface and bulk vapor is small, the caused mass transfer diffusion is significant, which should be treated carefully in both experimental and theoretical studies. The sensible heat transfer resistance is small in this case and may be neglected.

\section{References}

[1] Fronk, B.M. \& Garimella, S., In-tube condensation of zeotropic fluid mixtures: A review. International Journal of Refrigeration, 36(2), pp. 534561, 2013.

[2] Koyama, S., Yu, J. \& Ishibashi, A., Condensation of natural refrigerant mixtures in horizontal smooth tube - prediction model and calculation results. International Refrigeration and Air Conditioning Conference, Paper 432, pp. 331-336, 1998.

[3] Jin, D.X., Kwon, J. \& Kima, M., Prediction of in-tube condensation heat transfer characteristics of binary refrigerant mixtures. International Journal of Refrigeration, 26(5), pp. 593-600, 2003.

[4] Linton, W.H. \& Sherwood, T.K., Mass transfer from solid shapes to water in stream-line and turbulent flow. Chemical Engineering Progress, 46(5), pp. 258-264, 1950.

[5] Lemmon, E.W., Huber, M.L. \& McLinden, M.O., NIST Reference Fluid Thermodynamic and Transport Properties - REFPROP, Version 8.0. National Institute of Standards and Technology, Gaithersburg, MD, 2007.

[6] Friedel, L., Improved friction pressure drop correlations for horizontal and vertical two-phase pipe flow. European Two-phase Flow Group Meeting, Ispra, Italy, Paper E2, 1979.

[7] Haaland, S., Simple and explicit formulas for the skin friction in turbulent pipe flow. Journal Fluids Engineering, 105(1), pp. 89-90, 1983.

[8] Chisholm, D., An equation for velocity ratio in two-phase flow. NEL Report No 535, National Engineering Laboratory, Department of Trade and Industry, 1972.

[9] Dobson, M.K. \& Chato, J.C., Condensation in smooth horizontal tubes. Journal of Heat Transfer, 120(1), pp. 193-213, 1998.

[10] Jiang, B.N., The Least-Squares Finite Element Method: Theory and Applications in Computational Fluid Dynamics and Electromagnetics. Springer: New York, 1998. 
240 Advanced Computational Methods and Experiments in Heat Transfer XIII

[11] Kogawa, K., An experimental study on condensation of R134a/R123 mixtures inside horizontal smooth and micro-fin tubes. MS thesis, Kyushu University, Fukuoka, Japan, 1993. 